\title{
Preferência de Atta sexdens rubropilosa Forel (1908) (Hymenoptera: Formicidae) por espécies e híbridos de eucalipto
}

Este trabalho teve como objetivo avaliar a preferência de Atta sexdens rubropilosa Forel (1908) por diferentes espécies e híbridos de eucalipto, provenientes de diferentes localidades do estado do Mato Grosso. Foram coletadas amostras foliares de 21 tratamentos plantados nos municípios de Itiquira, Santa Rita do Trivelato e Sinop. Os testes de atratividade foram feitos em campo no município de Cuiabá (MT). Para os testes de atratividade, foram utilizadas placas de alumínio numeradas, sendo que cada espécie, clone ou híbrido foram considerados como tratamento. Em cada placa de alumínio, foram colocados 25 discos foliares de cada tratamento e as placas foram dispostas ao longo do carreador ativo dos formigueiros, sendo quatro repetições por tratamento. A atratividade foi avaliada, determinando-se a quantidade de discos foliares transportados dos tratamentos. As saúvas apresentaram maior preferência foliar pelos híbridos Eucalyptus urophyla $x$ Eucalyptus grandis (urograndis). Eucalyptus camaldulensis e Corymbia citriodora foram as espécies com menor preferência, podendo afirmar que espécies seminais apresentam menor preferência de carregamento pelas formigas em comparação com as clonais. As regiões onde os eucaliptos foram plantados afetam na preferência de carregamento foliar pelas formigas, sendo os situados em Santa Rita do Trivelato os que apresentaram maior preferência de carregamento.

Palavras-chave: Eucalyptus spp.; Formigas cortadeiras; Forrageamento; Povoamento florestal.

\section{Preference of Atta sexdens rubropilosa Forel (1908) (Hymenoptera: Formicidae) by eucalyptus species and hybrids}

\begin{abstract}
The objective of this work was to evaluate the preference of Atta sexdens rubropilosa Forel (1908) for different eucalyptus species and hybrids from different localities of the state of Mato Grosso. Leaf samples were collected from 21 treatments planted in the municipalities of Itiquira, Santa Rita do Trivelato and Sinop. The attractiveness tests were done in the field in Cuiabá (MT). For the attractiveness tests, numbered aluminum plates were used, and each species, clone or hybrid were considered as treatment. In each aluminum plate, 25 leaf discs of each treatment were placed and the plates were arranged along the anthill's active carrier, four replications per treatment. Attractiveness was evaluated by determining the amount of leaf disks transported from the treatments. The leaves presented higher leaf preference for the hybrids Eucalyptus urophyla x Eucalyptus grandis (urograndis). Eucalyptus camaldulensis and Corymbia citriodora were the species with the lowest preference, and it can be stated that seminal species have lower preference for carrying by ants compared to clonal ones. The regions where the eucalyptus were planted affect the leaf loading preference by the ants, and those located in Santa Rita do Trivelato showed the highest loading preference.
\end{abstract}

Keywords: Eucalyptus spp.; Leaf-cutting ants; Foraging; Forest settlement.

Topic: Ciências Florestais

Reviewed anonymously in the process of blind peer
Received: 05/06/2019

Approved: 02/07/2019
Joilson Onofre Pereira dos Santos

Universidade Federal de Mato Grosso, Brasil

http://lattes.cnpq.br/9021928521407718

joilson.eng@gmail.com

Otávio Peres Filho (iD

Universidade Federal de Mato Grosso, Brasil

http://lattes.cnpq.br/1754925579953049

http://orcid.org/0000-0002-6669-5939

peres@ufmt.br

Marcelo Dias de Souza (iD

Universidade de Cuiabá, Brasil

http://lattes.cnpq.br/9474670068485343

http://orcid.org/0000-0002-4348-4645

marcelo.dias@florestal.eng.br

\author{
Alberto Dorval (it) \\ Universidade Federal de Mato Grosso, Brasi \\ http://lattes.cnpq.br/2213033977376867 \\ http://orcid.org/0000-0001-8219-6294 \\ adorval@terra.com.br \\ Lilian Guimarães de Favare (id \\ Universidade Federal de Mato Grosso, Brasil \\ http://lattes.cnpq.br/7561645966079704 \\ http://orcid.org/0000-0002-6715-8000 \\ lilianfavare@hotmail.com
}

Referencing this:

SANTOS, J. O. P.; PERES FILHO, O.; SOUZA, M. D.; DORVAL, A.; FAVARE, L. G.. Preferência de Atta sexdens rubropilosa Forel (1908) (Hymenoptera: Formicidae) por espécies e híbridos de eucalipto. Revista Ibero-Americana de Ciências Ambientais, v.10, n.4, p.9-20, 2019. DOI: http://doi.org/10.6008/CBPC2179-6858.2019.004.0002 


\section{INTRODUÇÃO}

O gênero Eucalyptus é conhecido por sua grande variabilidade genética, em que apresenta centenas de espécies com diferentes propriedades físicas e químicas que fazem com que os eucaliptos sejam plantados para as mais diversas finalidades. Para Santana et al. (1990), em plantios de eucalipto podem ocorrer problemas que, muitas vezes, comprometem o sucesso do reflorestamento, tais como a presença de pragas na cultura. Dos diversos insetos-praga associados à cultura do eucalipto, destacam-se as formigas cortadeiras devido aos constantes ataques que essas formigas causam em diversas espécies e híbridos desse vegetal.

As formigas cortadeiras dos gêneros Atta (saúva) e Acromyrmex (quenquém) são consideradas pragas constantes nas diversas fases do desenvolvimento de um povoamento florestal. De acordo com Zanetti et al. (2002), o ataque realizado por formigas cortadeiras é de maneira intensa e constante, podendo acarretar danos em qualquer fase do desenvolvimento do vegetal, ocasionados por cortes de folhas, brotos, ramos finos e flores dos vegetais. De acordo com Quinlan et al. (1979), estes insetos necessitam do material de origem vegetal, pois servem de substrato para o crescimento do fungo Leucoagaricus gongylophorus (Möller) Singer que é uma de suas principais fontes de alimento. A preferência do corte das espécies vegetais está relacionada com a exigência nutricional do fungo simbionte (BASS et al., 1995; BORBA et al., 2006).

Vários autores realizaram trabalhos para quantificar os danos causados pelas formigas, a fim de se obter dados importantes para o manejo da espécie, além de fornecer subsídios para Manejo Integrado de Pragas (MIP) de formigas cortadeiras. Cantarelli et al. (2008), ao quantificar as perdas de Pinus após o ataque de formigas cortadeiras, verificaram que o ataque por estes insetos, no primeiro ano do plantio, afeta negativamente o diâmetro, a altura e o índice de produtividade, e caso o ataque ocorra no segundo ano, o ataque afeta negativamente os parâmetros altura e índice de produtividade. Já Bertulio (2008), ao avaliar desfolhas artificiais sucessivas em clones de híbridos Eucalyptus urophylla S.T. Blake x Eucalyptus grandis Hill ex-Maiden (urograndis) e. urophyla x Eucalyptus camaldulensis Dehnh (urocam), verificou que o volume calculado foram 191,14\% e 108,80\%, menores respectivamente em relação a testemunha.

Em ecossistema natural, as formigas mostram preferência por algumas espécies vegetais, as quais são constantemente desfolhadas, enquanto outras não são atacadas, embora sejam abundantes e localizadas próximas ao ninho (BORBA et al. 2006). De acordo com Nickele et al. (2013), algumas plantas apresentam resistência contra o ataque de formigas cortadeiras, da qual poderiam ser utilizados como uma alternativa de controle, entretanto, até o momento não são aplicáveis, pois necessitam de maiores pesquisas, tanto em nível de laboratório, como de campo. Lucia et al. (2008) relata sobre a importância de estudar plantas que apresentam resistência natural ao ataque de formigas cortadeiras no campo, sendo possível ainda a descoberta de plantas tóxicas as formigas ou para o fungo simbionte.

Peres Filho et al. (2002), estudando a preferência de carreamento de 42 espécies vegetais por Atta sexdens rubropilosa Forel, constatou que E. grandis teve menos disco foliares carregados em comparação com as demais espécies florestais nativas e exóticas, em condições de laboratório. Segundo Peres Filho et al. (2002), dependendo da diversidade de espécies florestais testadas, o comportamento das formigas pode ser 
alterado. Os autores ainda destacam que os compostos presentes nas folhas das diferentes espécies podem interferir na preferência de ataque pelas formigas cortadeiras.

Dessa forma, o estudo teve como objetivo avaliar a preferência de $A$. sexdens rubropilosa, por diferentes espécies, híbridos e clones de Eucalyptus provenientes de diferentes localidades do estado do Mato Grosso, em condições de campo, a fim de fornecer informações importantes sobre influência das localidades e os diferentes tratamentos em relação à preferência do carregamento foliar para o interior do formigueiro.

\section{MATERIAIS E MÉTODOS}

\section{Locais de coleta das folhas}

Para os testes de atratividade, foram coletadas duas espécies, 17 clones e dois híbridos de eucaliptos provenientes de três localidades distintas do estado de Mato Grosso (tabela 1). Todos os indivíduos amostrados foram plantados no período de novembro-2008 a março-2009, de acordo com projeto experimental da Associação dos Reflorestadores do Estado do Mato Grosso (AREFLORESTA), em que as árvores foram plantadas nos municípios de Dom Aquino, Chapada dos Guimarães, Sinop, Itiquira, Nobres, Cáceres e Santa Rita do Trivelato.

Tabela 1: Espécies, híbridos e clones de eucalipto utilizadas nos testes de preferência de Atta sexdens rubropilosa ao carregamento foliar em 2010.

\begin{tabular}{|c|c|}
\hline Espécie/Híbrido & Tipo de plantio \\
\hline Eucalyptus urophyla $\times$ Eucalyptus grandis (EUCA 103) & Clonal \\
\hline Eucalyptus urophyla x Eucalyptus grandis (EUCA 105) & Clonal \\
\hline Eucalyptus grandis x Eucalyptus resinífera (EUCA 608) & Clonal \\
\hline Eucalyptus camaldulensis (S23) & Clonal \\
\hline Eucalyptus urophyla $\times$ Eucalyptus grandis (H13) & Clonal \\
\hline Eucalyptus camaldulensis x Eucalyptus spp. (1277) & Clonal \\
\hline Eucalyptus urophyla x Eucalyptus grandis (Urograndis Acesita) & Seminal \\
\hline Eucalyptus urophyla $\mathrm{x}$ Eucalyptus grandis (GG100) & Clonal \\
\hline Eucalyptus urophyla $\times$ Eucalyptus grandis (IO42) & Clonal \\
\hline Eucalyptus urophyla x Eucalyptus grandis (Urograndis Luiz A.) & Seminal \\
\hline Corymbia citriodora (E. citriodora) & Seminal \\
\hline Eucalyptus camaldulensis (E. camaldulensis) & Seminal \\
\hline Eucalyptus urophyla $\times$ Eucalyptus grandis (F1H13) & Clonal \\
\hline Eucalyptus camaldulensis (F3C219) & Clonal \\
\hline Eucalyptus urophyla x Eucalyptus grandis (F8 Copener) & Clonal \\
\hline Eucalyptus urophyla x Eucalyptus grandis (F11 Copener) & Clonal \\
\hline Eucalyptus urophyla x Eucalyptus grandis (H77) & Clonal \\
\hline Eucalyptus urophyla x Eucalyptus grandis (I144) & Clonal \\
\hline Eucalyptus urophyla x Eucalyptus grandis (1224) & Clonal \\
\hline Urograndis $\mathrm{x}$ E. camaldulensis (VM 01) & Clonal \\
\hline Corymbia citriodora (LCA019) & Seminal \\
\hline
\end{tabular}

Para efeito de representatividade, foram escolhidos três locais para coleta do material e aplicação dos testes, sendo selecionados para coleta os plantios situados nos municípios de Itiquira, Santa Rita do Trivelato e Sinop, por serem os locais mais ao Sul $\left(170177^{\prime} 34,4^{\prime \prime} \mathrm{S} / 55010^{\prime} 22,8^{\prime \prime} \mathrm{W}\right)$, Centro $\left(14 \div 02^{\prime} 05,5^{\prime \prime} \mathrm{S} / 55^{\circ} 03^{\prime} 30,8^{\prime \prime} \mathrm{W}\right)$ e Norte $\left(11^{\circ} 51^{\prime} 59,9^{\prime \prime} \mathrm{S} / 55^{\circ} 27^{\prime} 58,7^{\prime \prime} \mathrm{W}\right)$, respectivamente, do estado de Mato Grosso. 
No solo do plantio situado no município de Itiquira, os percentuais de areia, silte e argila são de $87,7 \%, 3,2 \%$ e $9,1 \%$ respectivamente, sendo caracterizado como de textura areia franca. A região possui precipitação média anual de $1.500 \mathrm{~mm}$, com intensidade máxima de dezembro a fevereiro. Temperatura média anual de $22^{\circ} \mathrm{C}$, maior máxima $40^{\circ} \mathrm{C}$ e menor mínima $0^{\circ} \mathrm{C}$ (RESENDE et al. 2002; MARQUES, 2012).

No solo do plantio situado no município de Santa Rita do Trivelato possui percentuais de areia, silte e argila em $86,1 \%, 4,8 \%$ e 9,1\% respectivamente, sendo caracterizado como de textura areia franca. A região possui precipitação média anual de $2000 \mathrm{~mm}$, com intensidade máxima em janeiro, fevereiro e março. Temperatura média anual de $24^{\circ} \mathrm{C}$, maior máxima $38^{\circ} \mathrm{C}$ e menor $4^{\circ} \mathrm{C}$ (RESENDE et al., 2002; ROSA et al., 2007).

No solo do plantio situado no município de Sinop possui percentuais de areia, silte e argila em 47,6\%, $10,1 \%$ e $42,3 \%$ respectivamente, sendo caracterizado como de textura franco argilosa. A região possui precipitação média de $2500 \mathrm{~mm}$, com maior intensidade nos meses de janeiro, fevereiro e março. Temperatura média anual de $24^{\circ} \mathrm{C}$, maior máxima $40^{\circ} \mathrm{C}$ e menor $0^{\circ} \mathrm{C}$ (RESENDE et al.; 2002).

\section{Metodologia de coleta das folhas vegetais}

Nos locais de plantio foram implantados, de acordo com o projeto experimental da AREFLORESTA, quatro repetições de 49 árvores para cada tratamento, sendo coletado o material foliar do terço inferior da árvore central do primeiro bloco de cada tratamento. No caso de falhas, foi feito o caminhamento em sentido horário até que se encontrasse um exemplar vivo, segundo metodologia aplicada por Stradling (1978) (figura 1). As folhas coletadas foram mantidas em sacolas de polietileno e armazenadas em caixas térmicas contendo gelo para evitar alterações no conteúdo de umidade foliar. Além disso, entre o gelo e as sacolas com as folhas foi colocada uma pequena camada de folhas para evitar o contato direto do gelo com as folhas dos tratamentos.

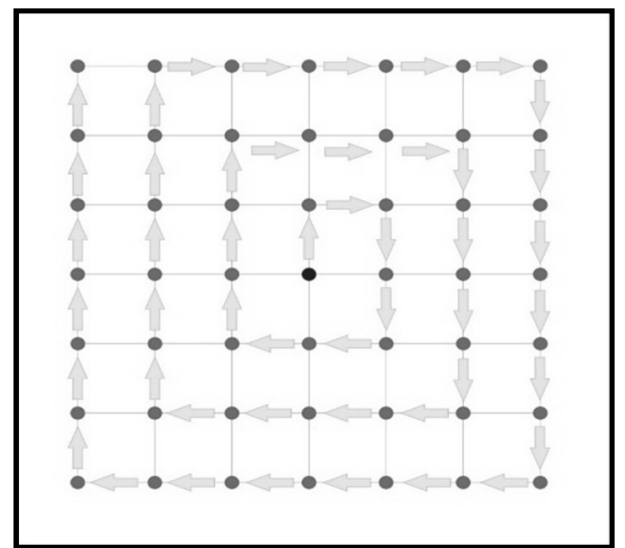

Figura 1: Diagrama esquemático de coleta de folhas vegetais em cada talhão dos tratamentos de 2010.

\section{Testes de atratividade}

O experimento foi realizado em condições de campo em outubro-2010, onde foi feito o teste de atratividade com formigueiro ativo de $A$. sexdens rubropilosa (saúva limão), com dimensões de 3,5m x 6,2m $\left(21,7 \mathrm{~m}^{2}\right)$ situado em um fragmento de cerrado localizado a 3,9km - Leste do campus da Universidade Federal de Mato Grosso (UFMT), no município de Cuiabá (MT). As coordenadas do formigueiro de teste são $15^{\circ} 37^{\prime}$ 
$39,77^{\prime \prime} \mathrm{S} / 56^{\circ} 01^{\prime} 57,40^{\prime \prime} \mathrm{W}$. Os testes de atratividade foram realizados no período noturno, uma vez que, de acordo com observações de campo, é o período de maior atividade das formigas para corte e transporte das folhas das espécies alvo. Para efeito de padronização, os testes de atratividade foram realizados entre as $18 \mathrm{~h}$ e $22 \mathrm{~h}$.

Foi utilizada a metodologia adaptada de Cherret et al. (1970), utilizando-se placas de alumínio de $15 \mathrm{~cm} \times 15 \mathrm{~cm}$ com 100 quadrículas de $1 \mathrm{~cm} \times 1 \mathrm{~cm}$ e bordaduras de $2,5 \mathrm{~cm}$ (figura $2 \mathrm{~A}$ ). As placas foram devidamente lavadas com sabão neutro e esterilizadas em estufa a $150^{\circ} \mathrm{C}$, pelo período de uma hora, antes e após qualquer utilização. As placas foram numeradas de um a 21 e cada espécie, híbrido e clone de eucalipto foi considerado como um tratamento. As amostras foliares foram obtidas utilizando um vazador metálico de forma circular, com diâmetro de 0,5cm (figura 2B).

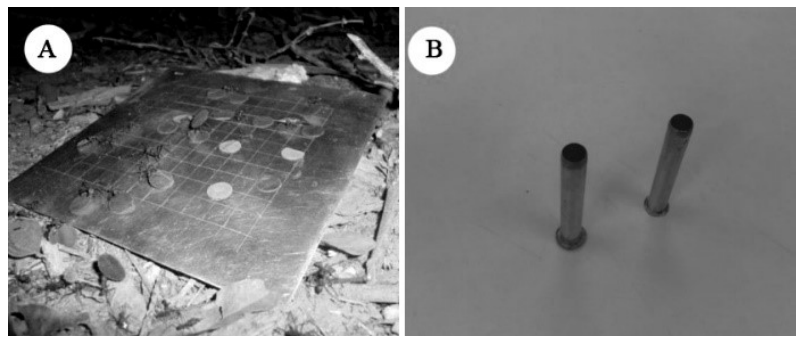

Figura 2: Placas de alumínio contendo 100 quadrículas para os discos foliares (A) e vazador metálico utilizado na confecção dos discos foliares.

Cada placa recebeu 25 discos foliares de cada tratamento, sendo dispostas a cinco centímetros de distância da trilha ativa do formigueiro de forma aleatória e ao acaso, das quais foram disponibilizados às saúvas por um período de 20 minutos ou até que a última amostra, de qualquer um dos tratamentos, tivesse sido carregada. Foram realizadas quatro repetições por tratamento, sendo observados por quatro dias. Para as análises, as repetições foram consideradas a média dos quatro dias para cada repetição.

\section{Análises dos Dados}

O delineamento foi inteiramente casualizado, sendo que foi analisada a preferência de $A$. sexdens rubropilosa por 17 clones, dois híbridos e duas espécies de eucalipto com dois anos de idade, ou seja, 21 tratamentos com quatro repetições, sendo uma análise para cada local de coleta. Para comparação entre os locais de coleta em relação aos tratamentos, os dados foram dispostos em esquema fatorial 18x3 (Tratamentos X Locais de coleta), em que não foi possível avaliar os 21 tratamentos, pois na localidade de Santa Rita do Trivelato, os clones: EUCA 608, H77 e 1224 não sobreviveram à seca prolongada no período de maio a setembro de 2010.

A variável avaliada foi correspondente a quantidade de disco foliar retirados das placas e transportados para o interior do formigueiro, sendo os dados submetidos à análise de variância (ANOVA) e as médias dos tratamentos comparadas pelo teste de Tukey ao nível de $5 \%$ de probabilidade de erro. Para a uniformização e normalidade dos dados, estes foram submetidos aos testes de normalidade (Kolmogorov D: Normal test) e homogeneidade de variância (Bartlett), das quais foram transformados pela fórmula $\sqrt{X+0,5}$ 
, conforme preconizado por Banzatto et al. (2006). As análises estatísticas foram efetuadas utilizando-se o software Sisvar (FERREIRA, 2008).

\section{RESULTADOS E DISCUSSÃO}

\section{Preferência de $A$. sexdens rubropilosa por discos foliares de eucaliptos provenientes de cada município}

As formigas apresentaram preferência diferenciada entre os tratamentos dos municípios avaliados, da qual foi constatado que as folhas provenientes da região de Santa Rita do Trivelato tiveram maiores percentuais de carregamento, sendo o clone 1277 (E. camaldulensis x Eucalyptus spp.) o que apresentou maior média percentual de carregamento foliar, 99\% (tabela 2). A maioria dos tratamentos dessa região apresentou percentual acima dos $74 \%$, apenas nos tratamentos puros de E. camaldulensis e C. citriodora que tiveram percentual de carregamento foliar abaixo dos $22 \%$.

Tabela 2: Percentual médio de discos foliares transportados dos diferentes materiais de eucaliptos do município de Santa Rita do Trivelato (MT), com dois anos de idade, carregados por Atta sexdens rubropilosa.

\begin{tabular}{|c|c|c|c|}
\hline \multirow{2}{*}{$\begin{array}{l}\text { Tratamentos } \\
\text { Eucalyptus camaldulensis x Eucalyptus spp. (1277) }\end{array}$} & \multirow{2}{*}{$\begin{array}{c}\text { Disco foliar (\%) } \\
99\end{array}$} & \multicolumn{2}{|c|}{ Medias $^{1} \pm$ Desvio padrão } \\
\hline & & $5,02 \pm 0,05$ & $\mathrm{a}$ \\
\hline Eucalyptus urophyla $\times$ Eucalyptus grandis (GG100) & 97 & $4,97 \pm 0,09$ & $\mathrm{a}$ \\
\hline Eucalyptus urophyla x Eucalyptus grandis (F1H13) & 96 & $4,94 \pm 0,08$ & $\mathrm{a}$ \\
\hline Eucalyptus urophyla $\times$ Eucalyptus grandis ( $\mathrm{H} 13)$ & 96 & $4,94 \pm 0,14$ & $\mathrm{a}$ \\
\hline Eucalyptus urophyla $\times$ Eucalyptus grandis (F8 Copener) & 95 & $4,92 \pm 0,19$ & $\mathrm{a}$ \\
\hline Eucalyptus urophyla x Eucalyptus grandis (EUCA 103) & 94 & $4,89 \pm 0,24$ & $\mathrm{a}$ \\
\hline Eucalyptus camaldulensis (S23) & 92 & $4,84 \pm 0,22$ & $\mathrm{a}$ \\
\hline Eucalyptus urophyla x Eucalyptus grandis (Urograndis Acesita) & 92 & $4,84 \pm 0,28$ & $\mathrm{a}$ \\
\hline Eucalyptus urophyla $\mathrm{x}$ Eucalyptus grandis (Urograndis Luiz A.) & 91 & $4,81 \pm 0,27$ & $\mathrm{a}$ \\
\hline Eucalyptus urophyla x Eucalyptus grandis (I144) & 88 & $4,71 \pm 0,68$ & $\mathrm{a}$ \\
\hline Corymbia citriodora (LCA019) & 83 & $4,59 \pm 0,44$ & $\mathrm{a}$ \\
\hline Eucalyptus urophyla x Eucalyptus grandis (IO42) & 83 & $4,55 \pm 0,78$ & $\mathrm{a}$ \\
\hline Eucalyptus urophyla x Eucalyptus grandis (F11 Copener) & 80 & $4,45 \pm 0,91$ & $\mathrm{a}$ \\
\hline Urograndis x Eucalyptus camaldulensis (VM 01) & 77 & $4,42 \pm 0,42$ & $\mathrm{a}$ \\
\hline Eucalyptus camaldulensis (F3C219) & 73 & $4,25 \pm 0,92$ & $\mathrm{a}$ \\
\hline Eucalyptus urophyla x Eucalyptus grandis (EUCA 105) & 74 & $4,21 \pm 1,26$ & $\mathrm{a}$ \\
\hline Corymbia citriodora (E. citriodora) & 22 & $2,26 \pm 0,36$ & $\mathrm{~b}$ \\
\hline Eucalyptus camaldulensis (E. camaldulensis) & 19 & $1,98 \pm 1,66$ & $\mathrm{C}$ \\
\hline p-value & - & \multicolumn{2}{|l|}{$0,000041^{* *}$} \\
\hline CV (\%) & - & \multicolumn{2}{|l|}{13,93} \\
\hline
\end{tabular}

Legenda: ${ }^{1}$ Dados transformados em $\sqrt{X+0,5} ;{ }^{* *}$ Significativo a 1\% de probabilidade de erro. Médias seguidas de mesma letra não diferem estatisticamente entre si ao nível de $5 \%$ de probabilidade pelo teste de Tukey.

A preferência A. sexdens rubropilosa aos discos foliares de Santa Rita do Trivelato foi maior no clone 1277, entretanto, não diferiu da maioria dos tratamentos, apenas $C$. citriodora e $E$ camaldulensis, ambos seminais, que apresentaram menor preferência. Lucia et al. (1995) relatou que, em condições de laboratório, as formigas cortadeiras do gênero Acromyrmex apresentam menor preferência a $C$. citriodora, pois foi observado menor transporte de fragmentos foliares dessa espécie no interior dos jardins de fungo em relação as demais espécies de eucalipto testadas.

Nas folhas provenientes da região de Itiquira, foi constatado que o clone 1277 obteve maior carregamento foliar (média de 71\%), entretanto, não diferiu da maioria dos tratamentos. Os tratamentos IO42, S23, EUCA 105, EUCA 608 e F3C219 apresentaram percentual médio de carregamento abaixo dos 36\% sendo considerados como os vegetais com menor preferência diferindo dos demais tratamentos (tabela 3). 
Tabela 3: Percentual médio de discos foliares dos diferentes materiais de eucaliptos. provenientes do município de Itiquira (MT), com dois anos de idade, carregados por Atta sexdens rubropilosa.

\begin{tabular}{|c|c|c|c|}
\hline Tratamentos & Disco foliar (\%) & Medias $^{1} \pm$ Desvio pa & drão \\
\hline Eucalyptus camaldulensis x Eucalyptus spp. (1277) & 71 & $4,11 \pm 1,34$ & $\mathrm{a}$ \\
\hline Eucalyptus urophyla $\times$ Eucalyptus grandis (F11 Copener) & 65 & $3,99 \pm 1,02$ & $\mathrm{a}$ \\
\hline Eucalyptus urophyla x Eucalyptus grandis (Urograndis Acesita) & 64 & $3,97 \pm 0,96$ & $\mathrm{a}$ \\
\hline Corymbia citriodora (LCA019) & 49,25 & $3,96 \pm 0,15$ & $\mathrm{a}$ \\
\hline Eucalyptus urophyla x Eucalyptus grandis (Urograndis Luiz A.) & 60 & $3,88 \pm 0,71$ & $\mathrm{a}$ \\
\hline Eucalyptus urophyla x Eucalyptus grandis (F8 Copener) & 60 & $3,77 \pm 1,30$ & $\mathrm{a}$ \\
\hline Eucalyptus urophyla $\times$ Eucalyptus grandis (EUCA 103) & 55 & $3,74 \pm 0,57$ & $\mathrm{a}$ \\
\hline Eucalyptus camaldulensis (E. camaldulensis) & 49 & $3,54 \pm 0,49$ & $\mathrm{a}$ \\
\hline Eucalyptus urophyla $\mathrm{x}$ Eucalyptus grandis (I144) & 52 & $3,50 \pm 1,28$ & $\mathrm{a}$ \\
\hline Urograndis $\mathrm{x}$ Eucalyptus camaldulensis (VM 01) & 51 & $3,47 \pm 1,26$ & $\mathrm{a}$ \\
\hline Corymbia citriodora (E. citriodora) & 45 & $3,42 \pm 0,07$ & $\mathrm{a}$ \\
\hline Eucalyptus urophyla $\times$ Eucalyptus grandis (GG100) & 47 & $3,11 \pm 1,83$ & $\mathrm{a}$ \\
\hline Eucalyptus urophyla x Eucalyptus grandis (1224) & 38 & $2,99 \pm 1,18$ & $\mathrm{a}$ \\
\hline Eucalyptus urophyla x Eucalyptus grandis (F1H13) & 37 & $2,92 \pm 1,26$ & $a b$ \\
\hline Eucalyptus urophyla x Eucalyptus grandis (H13) & 38 & $2,82 \pm 1,63$ & $a b$ \\
\hline Eucalyptus urophyla x Eucalyptus grandis (H77) & 33 & $2,82 \pm 1,01$ & $a b$ \\
\hline Eucalyptus urophyla x Eucalyptus grandis (IO42) & 36 & $2,78 \pm 1,52$ & $\mathrm{~b}$ \\
\hline Eucalyptus camaldulensis (S23) & 34 & $2,75 \pm 1,36$ & $\mathrm{~b}$ \\
\hline Eucalyptus urophyla $\mathrm{x}$ Eucalyptus grandis (EUCA 105) & 31 & $2,69 \pm 1,13$ & $\mathrm{~b}$ \\
\hline Eucalyptus grandis x Eucalyptus resinifera (EUCA 608) & 28 & $2,67 \pm 0,69$ & $\mathrm{~b}$ \\
\hline Eucalyptus camaldulensis (F3C219) & 21 & $2,38 \pm 0,25$ & $\mathrm{~b}$ \\
\hline p-value & - & \multicolumn{2}{|l|}{$0,038^{*}$} \\
\hline CV (\%) & - & \multicolumn{2}{|l|}{30,94} \\
\hline
\end{tabular}

Legenda: ${ }^{1}$ Dados transformados em $\sqrt{X+0,5} ;{ }^{*}$ Significativo a $5 \%$ de probabilidade de erro. Médias seguidas de mesma letra não diferem estatisticamente entre si ao nível de $5 \%$ de probabilidade pelo teste de Tukey.

Dos 13 tratamentos com maiores médias significativas de transporte, dez apresentam genes características do híbrido urograndis (E. urophyla x E. grandis), cabendo ressaltar que esta preferência ocorre em comparação com os demais tratamentos avaliados da mesma região, demonstrando que Itiquira apresentam condiçoes aos hibridos de urocam que favorecem na escolha por $A$. sexdens rubropilosa. As folhas provenientes da região de Sinop apresentaram percentuais médios de carregamento foliar abaixo de $50 \%$, pois os tratamentos que apresentaram maior quantidade de material transportado foram os clones I224 e F3C219, ambos com 48\%, e ainda não diferindo da maioria dos tratamentos, exceto C. citriodora que obteve o menor carregamento foliar (tabela 4).

Tabela 4: Percentual médio de discos foliares dos diferentes materiais de Eucaliptos provenientes do município de Sinop (MT), com dois anos de idade, carregados por Atta sexdens rubropilosa.

\begin{tabular}{|c|c|c|c|}
\hline Tratamentos & Disco foliar (\%) & Medias $^{1} \pm$ Desvio $p$ & Urão \\
\hline Eucalyptus urophyla x Eucalyptus grandis (1224) & 48 & $3,49 \pm 0,18$ & $\mathrm{a}$ \\
\hline Eucalyptus camaldulensis (F3C219) & 48 & $3,48 \pm 1,06$ & $\mathrm{a}$ \\
\hline Eucalyptus urophyla x Eucalyptus grandis (F1H13) & 42 & $3,20 \pm 1,01$ & $a b$ \\
\hline Eucalyptus urophyla x Eucalyptus grandis $(\mathrm{H} 77)$ & 42 & $3,16 \pm 1,13$ & $a b$ \\
\hline Eucalyptus urophyla x Eucalyptus grandis ( $\mathrm{H} 13)$ & 37 & $3,04 \pm 0,78$ & $a b$ \\
\hline Eucalyptus camaldulensis x Eucalyptus spp. (1277) & 27 & $2,57 \pm 0,91$ & $a b$ \\
\hline Eucalyptus grandis x Eucalyptus resinifera (EUCA 608) & 27 & $2,55 \pm 0,99$ & $a b$ \\
\hline Eucalyptus urophyla x Eucalyptus grandis (IO42) & 24 & $2,52 \pm 0,41$ & $a b$ \\
\hline Eucalyptus urophyla $\times$ Eucalyptus grandis (EUCA 103) & 24 & $2,48 \pm 0,64$ & $a b$ \\
\hline Eucalyptus urophyla x Eucalyptus grandis (Urograndis Luiz A.) & 26 & $2,45 \pm 1,12$ & $a b$ \\
\hline Eucalyptus urophyla x Eucalyptus grandis (Urograndis Acesita) & 21 & $2,27 \pm 0,89$ & $a b$ \\
\hline Eucalyptus urophyla $\mathrm{x}$ Eucalyptus grandis (I144) & 23 & $2,24 \pm 1,26$ & $a b$ \\
\hline Eucalyptus urophyla $\times$ Eucalyptus grandis (EUCA 105) & 17 & $2,15 \pm 0,41$ & $a b$ \\
\hline Eucalyptus urophyla x Eucalyptus grandis (GG100) & 17 & $2,13 \pm 0,51$ & $a b$ \\
\hline Corymbia citriodora (LCA019) & 17 & $2,12 \pm 0,57$ & $a b$ \\
\hline Eucalyptus urophyla x Eucalyptus grandis (F8 Copener) & 15 & $1,91 \pm 0,91$ & $a b$ \\
\hline Urograndis $\mathrm{x}$ Eucalyptus camaldulensis (VM 01) & 12 & $1,76 \pm 0,72$ & $a b$ \\
\hline Eucalyptus urophyla $\mathrm{x}$ Eucalyptus grandis (F11 Copener) & 11 & $1,71 \pm 0,63$ & $a b$ \\
\hline Eucalyptus camaldulensis (E. camaldulensis) & 12 & $1,69 \pm 0,91$ & $a b$ \\
\hline Eucalyptus camaldulensis (S23) & 11 & $1,56 \pm 1,02$ & $a b$ \\
\hline Corymbia citriodora (E. citriodora) & 7 & $1,41 \pm 0,59$ & $\mathrm{~b}$ \\
\hline
\end{tabular}




\begin{tabular}{|c|c|c|}
\hline p-value & - & $0,0009^{* *}$ \\
\hline CV (\%) & - & 29,60 \\
\hline
\end{tabular}

Legenda: ${ }^{1}$ Dados transformados em $\sqrt{X+0,5} ;{ }^{* *}$ Significativo a 1\% de probabilidade de erro. Médias seguidas de mesma letra não diferem estatisticamente entre si ao nível de $5 \%$ de probabilidade pelo teste de Tukey.

Os discos foliares de $C$. citriodora foram os que apresentaram a menor quantidade de material carregado para o interior do ninho, com um percentual de 7\%, diferindo de todos os tratamentos. A não preferência de $A$. sexdens rubropilosa por C. citriodora já foi observado por Vendramin et al. (1995), ao avaliar a preferência desta espécie em comparação com E. camaldulensis, E. dunnii, E. grandis, E. saligna, E. torrelliana e E. urophylla. Na avaliação de Vendramin et al. (1995), C. citriodora apresentou maior grau de não preferência, seguido por E. grandis, enquanto as demais foram carregadas igualmente entre si pelas saúvas.

Os clones apresentam diversas características superiores a plantios seminais, tais como crescimento, propriedades químicas, largura e espessura da parede celular, propriedades físicas e mecânicas da madeira, qualidade da madeira para fins energéticos, energia e celulose (BOTREL et al., 2007; TRUGILHO et al., 2007). Entretanto, no presente trabalho, os eucaliptos seminais foram os que apresentaram menor preferência de carregamento foliar pelas formigas cortadeiras em comparação aos clonais.

Uma hipótese que pode explicar a baixa preferência ao corte por algumas espécies de eucalipto pode estar relacionada com os compostos secundários com ação repelente presentes nos óleos essenciais de algumas procedências de eucaliptos (MARSARO JÚNIOR et al., 2007). Os óleos essenciais de eucalipto são ricos em terpenoides que podem atuar como repelente as formigas cortadeiras, pois as formigas quando expostas ao óleo essencial, acarretam alterações de comportamento (MARSARO JÚNIOR et al., 2004).

Rezende et al. (1999) afirmou que folhas contendo alto teor de óleos essenciais e ainda mudas apresentando alta pilosidade nas folhas, podem servir como uma barreira ao corte e carregamento pelas formigas cortaderas, sendo essa uma das caracteristicas de C. citriodora. Pereira et al. (2006) afirmaram que folhas de $E$. camaldulensis e $C$. citriodora são ricos em óleos essenciais.

Hebling et al. (2000), estudando o efeito de Ipomoea batatas (L.) (batata-doce) em colonias de formigas cortadeiras, pôde constatar mortalidade de operárias e redução na quantidade de fungo simbionte produzido pelas formigas. Hebling et al. (2000) reforça a ideia que as folhas de batata-doce apresentam metabólitos secundários com ação tóxica as formigas por contato e/ou ingestão de seiva foliar. Vale ressaltar que variedades de eucalipto apresentam diferentes características vegetais, tais como textura, pilosidade e a dureza do material vegetal da folha ou partes vegetais como ponteiro e ramos, sendo essas as principais características que influenciam as formigas cortadeiras na preferência de corte (CHERRETT, 1972; HUBBELL et al., 1984).

Santana et al. (1989) reforça a ideia que, quanto maior o número de procedências testadas, mais evidentes se tornam as diferenças ou semelhanças entre as procedencias. De acordo com Lucia et al. (1995), espécies de Eucalyptus spp. contêm compostos secundários, em diferentes teores, e apresentam características físicas diversas em suas folhas, é de se esperar diferenças na preferencia de ao corte pelas formigas cortadeiras. 
Preferência de A. sexdens rubropilosa por discos foliares de eucaliptos em relação às regiões das coletas

Analisando conjuntamente os municípios, pode se constatar que os vegetais provenientes da região de Santa Rita do Trivelato foram os que apresentaram maiores quantidades de discos foliares transportados pelas formigas cortadeiras, seguidos de Itiquira e Sinop. Houve interação significativa entre os tratamentos e locais de coleta dos discos foliares, pois alguns tratamentos tiveram media diferenciada entre as regiões avaliadas, como observado no hibrido 1277, que obteve as maiores quantidades de discos foliares na região de Santa Rita do Trivelato e Itiquica (tabela 5).

Tabela 5: Análise fatorial do percentual médio de discos foliares dos diferentes materiais de Eucaliptos provenientes de Santa Rita do Tivelato, Itiquira e Sinop, carregados pelas formigas.

\begin{tabular}{|c|c|c|c|c|c|c|c|c|c|}
\hline \multirow{3}{*}{$\begin{array}{l}\text { Tratamentos } \\
\text { Eucalyptus spp. (1277) }\end{array}$} & \multicolumn{9}{|c|}{ Medias $^{1} \pm$ Desvio padrão dos municípios } \\
\hline & \multicolumn{3}{|c|}{ Itiquira } & \multicolumn{3}{|c|}{ Sinop } & \multicolumn{3}{|c|}{ Trivelato } \\
\hline & $4,11 \pm 1,34$ & $\mathrm{a}$ & $A B$ & $2,57 \pm 0,91$ & $a b$ & $\mathrm{~B}$ & $5,02 \pm 0,05$ & $\mathrm{a}$ & A \\
\hline Urograndis $\mathrm{x}$ Eucalyptus camaldulensis (VM 01) & $3,47 \pm 1,26$ & $\mathrm{a}$ & $A B$ & $1,76 \pm 0,72$ & $a b$ & $\mathrm{~B}$ & $4,42 \pm 0,42$ & $a b$ & A \\
\hline Eucalyptus urophyla $\mathrm{x}$ Eucalyptus grandis (Urograndis Luiz A.) & $3,88 \pm 0,71$ & $\mathrm{a}$ & A & $2,45 \pm 1,12$ & $a b$ & $\mathrm{~B}$ & $4,81 \pm 0,27$ & $\mathrm{a}$ & A \\
\hline Eucalyptus urophyla $\mathrm{x}$ Eucalyptus grandis (Urograndis Acesita) & $3,97 \pm 0,96$ & $\mathrm{a}$ & A & $2,27 \pm 0,89$ & $a b$ & $\mathrm{~B}$ & $4,84 \pm 0,28$ & $\mathrm{a}$ & A \\
\hline Eucalyptus camaldulensis (S23) & $2,75 \pm 1,36$ & $\mathrm{~b}$ & $\mathrm{~B}$ & $1,56 \pm 1,02$ & $\mathrm{~b}$ & $\mathrm{~B}$ & $4,84 \pm 0,22$ & $\mathrm{a}$ & A \\
\hline Corymbia citriodora (LCA019) & $3,96 \pm 0,15$ & $\mathrm{a}$ & A & $2,12 \pm 0,57$ & $a b$ & $\mathrm{~B}$ & $4,59 \pm 0,44$ & $a b$ & A \\
\hline Eucalyptus urophyla $\mathrm{x}$ Eucalyptus grandis (IO42) & $2,78 \pm 1,52$ & $a b$ & $\mathrm{~B}$ & $2,52 \pm 0,41$ & $a b$ & $\mathrm{~B}$ & $4,55 \pm 0,78$ & $a b$ & A \\
\hline Eucalyptus urophyla $\times$ Eucalyptus grandis (I144) & $3,50 \pm 1,28$ & $\mathrm{a}$ & A & $2,24 \pm 1,26$ & $a b$ & B & $4,71 \pm 0,68$ & $\mathrm{a}$ & A \\
\hline Eucalyptus urophyla $\times$ Eucalyptus grandis (H13) & $2,82 \pm 1,63$ & $a b$ & $\mathrm{~B}$ & $3,04 \pm 0,78$ & $a b$ & $\mathrm{~B}$ & $4,94 \pm 0,14$ & $\mathrm{a}$ & A \\
\hline Eucalyptus urophyla $\times$ Eucalyptus grandis (GG100) & $3,11 \pm 1,83$ & $\mathrm{a}$ & $\mathrm{B}$ & $2,13 \pm 0,51$ & $a b$ & $\mathrm{~B}$ & $4,97 \pm 0,09$ & $\mathrm{a}$ & A \\
\hline Eucalyptus urophyla $\times$ Eucalyptus grandis (F8 Copener) & $3,77 \pm 1,30$ & $\mathrm{a}$ & A & $1,91 \pm 0,91$ & $a b$ & $\mathrm{~B}$ & $4,92 \pm 0,19$ & $\mathrm{a}$ & A \\
\hline Eucalyptus camaldulensis (F3C219) & $2,38 \pm 0,25$ & $\mathrm{~b}$ & $\mathrm{~B}$ & $3,48 \pm 0,66$ & $\mathrm{a}$ & $A B$ & $4,25 \pm 0,92$ & $a b$ & A \\
\hline Eucalyptus urophyla x Eucalyptus grandis (F1H13) & $2,92 \pm 1,26$ & $a b$ & $\mathrm{~B}$ & $3,20 \pm 1,01$ & $a b$ & $\mathrm{~B}$ & $4,94 \pm 0,08$ & $\mathrm{a}$ & A \\
\hline Eucalyptus urophyla $\times$ Eucalyptus grandis (F11 Copener) & $3,99 \pm 1,02$ & $\mathrm{a}$ & $\mathrm{A}$ & $1,71 \pm 0,63$ & $a b$ & $\mathrm{~B}$ & $4,45 \pm 0,91$ & $a b$ & A \\
\hline Eucalyptus urophyla $\mathrm{x}$ Eucalyptus grandis (EUCA 105) & $2,69 \pm 1,13$ & $\mathrm{~b}$ & $\mathrm{AB}$ & $2,15 \pm 0,41$ & $a b$ & $\mathrm{~B}$ & $4,21 \pm 1,26$ & $a b$ & A \\
\hline Eucalyptus urophyla $\mathrm{x}$ Eucalyptus grandis (EUCA 103) & $3,74 \pm 0,57$ & $\mathrm{a}$ & $A B$ & $2,48 \pm 0,64$ & $a b$ & $\mathrm{~B}$ & $4,89 \pm 0,24$ & $\mathrm{a}$ & A \\
\hline Eucalyptus camaldulensis (E. camaldulensis) & $3,54 \pm 0,49$ & $\mathrm{a}$ & A & $1,69 \pm 0,91$ & $a b$ & $\mathrm{~B}$ & $2,26 \pm 0,36$ & bc & $A B$ \\
\hline Corymbia citriodora (E. citriodora) & $3,42 \pm 0,07$ & $\mathrm{a}$ & A & $1,41 \pm 0,59$ & $\mathrm{~b}$ & $\mathrm{~B}$ & $1,98 \pm 1,66$ & c & $B$ \\
\hline $\mathrm{p}$-value & \multicolumn{9}{|c|}{$0,00001^{* *}$} \\
\hline CV (\%) & \multicolumn{9}{|c|}{23,36} \\
\hline
\end{tabular}

Legenda: ${ }^{1}$ Dados transformados em $\sqrt{X+0,5} ;{ }^{* *}$ Significativo a 1\% de probabilidade de erro. Médias seguidas de mesma letra minúsculas nas colunas e maiúsculas nas linhas não diferem estatisticamente entre si ao nível de $5 \%$ de probabilidade pelo teste de Tukey.

A maioria dos tratamentos provenientes de Santa Rita do Trivelato apresentaram maiores medias percentuais de carregamento, diferindo das demais regiões, exceto para os tratamentos E. camaldulensis e C. citriodora que obtiveram maiores quantidades de discos foliares carregados na região de Itiquira. Chrispim et al. (2007) afirmou que a não preferência dos vegetais pelos insetos pode estar relacionada aos fatores climáticos e edáficos que afetam no desenvolvimento do vegetal, tais como unidade, temperatura e disponibilidade de nutrientes, sais minerais e até mesmo plantas adjacentes presentes ao plantio. Chrispim et al. (2007) ainda enfatiza que esses fatores podem contribuir para a manifestação de resistência nos vegetais, estabelecendo um microclima favorável ou desfavorável para o inseto em que pode atenuar ou inibir o ataque respectivamente.

Um fator que pode explicar o fato dos clones apresentarem média de carregamentos diferenciados entre as regiões avaliadas se dá pelas condições ambientais característicos de cada região, da qual afeta nas propriedades químicas e físicas dos vegetais afetando na preferência dos insetos, fato este constatado visualmente nas regiões de coletas dos discos foliares (figura 3). 


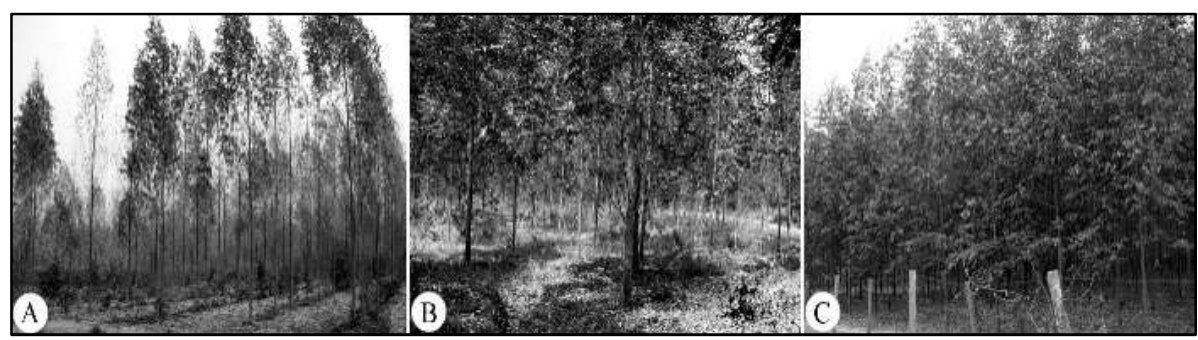

Figura 3: Locais de Coleta dos discos foliares dos tratamentos. A) Santa Rita do Trivelato; B) Itiquira; e C) Sinop.

De acordo com Ortiz (2017), os padrões de forrageamento podem estar ligados à sazonalidade, que no caso de formigas cortadeiras, apresentam maior atividade de forrageamento no período chuvoso, devido a maior e a melhor oferta de material verde coincidir nesse período. As chuvas promovem alterações no balanço químico dos vegetais, provocando respostas diferenciadas sobre a atividade de corte (LUCIA, 1993; DIEHL-FLEIG, 1995).

Em Santa Rita do Trivelato houve mortalidade de eucaliptos em detrimento as condições de estiagem, comprovando que as plantas passaram por condições de estresse hídrico. Esta cidade é destaque em Mato Grosso como uma das cidades que apresentam maior área reflorestada do estado, principalmente com plantios de Eucalyptus spp. (ROTTA 2009), da qual demonstra que a cidade necessita de constante combate, pois apresenta muitos plantios de eucalipto e ainda condições ambientais desfavoráveis aos vegetais, que pode ocasionar na preferência das folhas pelas formigas cortadeiras.

Nos plantios situados em Itiquira houve também mortalidade de alguns tratamentos, entretanto esta mortalidade ocorreu em detrimento ao ataque de Glycaspis brimblecombei Moore (psilídeo-de-concha) que é constante na região e ainda associado à estiagem que ocorre no município. Para Dahlsten (1999), a infestação do psilídeo-de-concha nas árvores acarreta no estresse do vegetal, da qual as tornam susceptíveis ao ataque de outras pragas, além de ocorrência de doenças.

De acordo com Santos et al. (1998), a deficiência hídrica em plantas cultivadas afeta o crescimento e o desenvolvimento das culturas provocando alterações no comportamento vegetal, tornando-a susceptível a ataque organismos, cuja irreversibilidade vai depender do genótipo, da duração, da severidade e do estágio de desenvolvimento da planta. Vale ressaltar que a região de Sinop apresenta os maiores índices pluviométricos, da qual não sofre com deficiência hídrica e ainda o plantio apresentou visualmente as melhores condições fitossanitárias, com baixa mortalidade dos vegetais e maiores densidade populacionais, em comparação as demais regiões.

\section{CONCLUSÕES}

Plantios florestais com clone 1277 ou híbridos de E. urophyla $x$ E. grandis necessitam de monitoramentos constante com intuito de identificar ataque de formigas cortadeiras para controle, principalmente nos locais plantios com estiagem prolongada. Espécies seminais apresentam maior resistência ao forrageamento de Atta sexdens rubropilosa, quando comparado com híbridos clonais, principalmente plantio seminal de Corymbia citriodora. 


\section{REFERÊNCIAS}

BANZATTO, D. A.; KRONKA, S. N.. Experimentação agrícola. 4 ed. Jaboticabal: Funep, 2006.

BASS, M.; CHERRETT, J. M.. Fungal hyphae as a source of nutrients for the leaf-cutting ant Atta sexdens. Physiological Entomology, v.20, p.1-6, 1995.

BERTULIO, V. G.. Efeitos da desfolha artificial no crescimento de plantas de Eucalyptuss spp. em uma área de reflorestamento, município de campo verde, estado de mato grosso. Dissertação (Mestrado em Ciências Florestais e Ambientais) - Universidade Federal de Mato Grosso, Cuiabá, 2008.

BORBA, R. S.; LOECKI, A. E.; BANDEIRA, J. M.; MORAES, C. L.; CENTENARO E. D.. Crescimento do fungo simbionte de formigas cortadeiras do gênero Acromyrmex em meios de cultura com diferentes extratos. Ciência Rural, v.36, n.3, p.725-730, 2006.

BOTREL, M. C. G.; SILVA, J. R. M.; TRUGILHO, P. F.; ROSADO, S. C. S.; FERNANDES, B. R.. Ganho genético em propriedades físicas e mecânicas de clones de Eucalyptus. Scientia Forestalis, n.76, p.13-19, 2007.

CANTARELLI, E. B.; COSTA E. C.; PEZZUTTI, R.; OLIVEIRA L. S.. Quantificação das perdas no desenvolvimento de Pinus taeda após o ataque de formigas cortadeiras. Ciência Florestal, v.18, n.1, p.39-45, 2008.

CHERRET, J. M.; SEAFORTH, C. E.. Phytochemical arrestants for leaf-cutting ants, Atta cephalotes (L.) and Acromyrmex octospinosus (Reich), with some notes on the ants responses. Bulletin of Entomological Research, v.59, p.615625, 1970.

CHERRETT, J. M.. Some factors involved in the selection of vegetable substrate by Atta cephalotes (L.) (Hymenoptera: Formicidae) in tropical rain forest. Journal of Animal Ecology, v.41, p.647-60, 1972.

CHRISPIM, T. P.; RAMOS, J. M.. Revisão de literatura: resistência de plantas a insetos. Revista Científica Eletrônica de Engenharia Florestal, v.10, 2007.

DAHLSTEN, D. L.. Biological control of the red gum lerp psyllid, a pest of Eucalyptus species in California. The regents of the University of California. Berkeley: 1999.

DIEHL-FLEIG, E.. Formigas: organização social e ecologia comportamental. São Leopoldo: UNISINOS, 2005.

FERREIRA, D. F. SISVAR: um programa para análises e ensino de estatística. Symposium, Lavras, v.6, p.36-41, 2008.

HEBLING, M. J. A.; BUENO, O. C.; MAROTI, P. S.; PAGNOCCA, F. C.; SILVA O. A.. Effects of leaves of Ipomoea batatas (Convolvulaceae) on nest development and on respiratory metabolism of leaf-cutting ants Atta sexdens L. (Hym., Formicidae). Journal of Applied Entomology, Berlin, v.124, p.249-252, 2000.

HUBBELL, S. P.; HOWARD, J. J.; WIEMER, D. F.. Chemical leaf repellency to an attine ant: seasonal distribution among potential host plant species. Ecology, v.65, n.4, p.1067-1076, 1984.

LUCIA, T. M. C. D.; MOREIRA, D. D. O.. Caracterização dos ninhos, In: LUCIA, T. M. C. D.. As formigas cortadeiras. Viçosa: Folha de Viçosa, 1993.

LUCIA, T. M. C.; MARINHO, C. G. S.; RIBEIRO, M. M. R.. Perspectiva no manejo de formigas cortadeiras. In: VILELA, E. F.; SANTOS, I. A.; SCHOEREDER, J. H.; SERRÃO, J. E.; CAMPOS, L. A. O.; LINO-NETO, J.. Insetos sociais: da biologia a aplicação. Viçosa: UFV, 2008. p.371-380.

LUCIA, T. M. C.; OLIVEIRA, M. A.; ARAÚJO, M. S.; VILELA, E. F.. Avaliação da não-preferência da formiga cortadeira Acromyrmex subterraneus Forel ao corte de Eucalyptus. Árvore, v.19, n.1, p.92-99, 1995.

MARQUES, M. D.. História do município de Itiquira. Itiquira: 2012.

MARSARO JÚNIOR, A. L.; RUGAMA, A. J. M.; LIMA, C. A.; LUCIA, T. M. C.. Preferência de corte de Eucalyptus spp. por Acromyrmex laticeps nigrosetosus Forel, 1908 (Hymenoptera: Formicidae) em condições de laboratório. Ciência Florestal, Santa Maria, v.17, n.2, p.171-174, 2007.

MARSARO JÚNIOR, A. L.; SOUZA, R. C.; LUCIA, T. M. C.. Behavioral changes in workers of the leaf-cutting ant Atta sexdens rubropilosa induced by chemical components of Eucalyptus maculata leaves. Journal of Chemical Ecology, Heidelberg, v.30, n.9, p.1771-1780, 2004.

NICKELE, M. A.; PIE, M. R.; REIS FILHO, W.; PENTEADO, S. R. C.. Formigas cultivadoras de fungos: estado da arte $\mathrm{e}$ direcionamento para pesquisas futuras. Pesquisa Florestal Brasileira, v.33, n.73, p.53-72, 2013.

ORTIZ, A. G.; PERES FILHO, O.; SANTOS, A.; SOUZA, M. D.; FAVARE, L. G.; NASCIMENTO, D. A.. Resposta do forrageamento de Acromyrmex rugosus (Smith, 1858) e Acromyrmex balzani (Emery, 1890) (Hymenoptera: Formicidae) a mudas de Eucalyptus camaldulensis Dehnh. Com diferentes restrições nutricionais. Espacios, v.38, n.544, p.1-10, 2017.

PEREIRA, L. G. B.; FERNANDES, J. B.; SILVA, M. F. G. F.; VIEIRA, P. C.; BUENO, O. C; CORRÊA, A. G..

Electrophysiological Responses of Atta sexdens rubropilosa Workers to Essential Oils of Eucalyptus and its Chemical Composition. Verlag der Zeitschrift für Naturforschung, Tübingen, v.61, n.9-10, p.749-755, 2006.

PERES FILHO, O.; DORVAL, A.; BERTI FILHO, E.. Preferência de saúva limão, Atta sexden srubropilosa forel, 1908 (Hymenoptera, Formicidae) a diferentes espécies florestais, em condições de laboratório. Ciência Florestal, Santa Maria, v.12, n.2, p.1-7, 2002.

QUINLAN, R. J.; CHERRETT, J. M.. The role of fungus in the diet of the leaf-cutting ant Atta cephalotes. Ecological entomology, v.4, p.151-160, 1979. 
RESENDE, M.; CURI, N.; REZENDE, S. B.; CORRÊA, G. F.. Pedologia: Base para distinção de ambientes. 4 ed. Viçosa: NEPUT, 2002.

REZENDE, M. A. A.; LUCIA, T. M. C.. Is Eucalyptus citriodora attacked by leaf-cutting ants?. Árvore, Viçosa, v.23, n.1, p.69-74, 1999.

ROSA, D. B.; SOUZA, R. R.; NASCIMENTO, L. A.; TOLEDO, L. G.; TOPANOTTI, D. Q.; NASCIMENTO, J. A.. A distribuição espacial das chuvas na porção centro oeste do estado de Mato Grosso - Brasil. Revista Eletrônica da Associação Dos Geógrafos Brasileiros, Três Lagoas, v.1, n.5, p.127-152, 2007.

ROTTA, G. W.. Desenvolvimento de duas espécies arbóreas e atributos químicos e físicos do solo em área de reflorestamento na Amazônia meridional. Dissertação (Mestrado em Ciências Ambientais) - Universidade do Estado de Mato Grosso, Cáceres, 2009.

SANTANA, D. L. Q.; ANJOS, N.. Resistência de Eucalyptus spp. (Myrtaceae) à Atta sexdens rubropilosa e Atta laevigata (Hymenoptera: Formicidae). Árvore, v.13, n.2, p.174-181, 1989.
SANTANA, D. L. Q.; COUTO, L.. Resistência intra-específica de eucaliptos a formigas-cortadeiras. Boletim de Pesquisa Florestal, n.20, p.13-21, 1990.

SANTOS, R. F.; CARLESSO, R.. Déficit hídrico e os processos morfológico e fisiológico das plantas. Revista Brasileira de Engenharia Agrícola e Ambiental, v.2, n.3, p.287-294, 1998.

STRADLING, D. J.. The influence of size on foraging in the ant, Atta cephalotes, and the effects of some plant defense mechanism. Journal of Animal Ecology, London, v.47, p.173188, 1978.

TRUGILHO, P. F.; BIANCHI, M. L.; ROSADO, S. C. S.; LIMA, J. T.. Qualidade da madeira de clones de espécies e híbridos naturais de Eucalyptus. Scientia Forestalis. v.73, p.55-62, 2007.

VENDRAMIM, J. D.; SILVEIRA NETO, S.; CERIGNONI, J. A.. Não-preferência de Atta sexdens rubropilosa Forel (i: Formicidae) por espécies de Eucalyptus. Ecossistema, v.20, n.1, p.87-92, 1995.

ZANETTI, R.; CARVALHO, G. A.; SANTOS, A.; SILVA, A S.; GODOY, M. S.. Manejo integrado de formigas cortadeiras. Lavras: UFLA, 2002.

A CBPC - Companhia Brasileira de Produção Científica (CNPJ: 11.221.422/0001-03) detém os direitos materiais desta publicação. Os direitos referem-se à publicação do trabalho em qualquer parte do mundo, incluindo os direitos às renovações, expansões e disseminações da contribuição, bem como outros direitos subsidiários. Todos os trabalhos publicados eletronicamente poderão posteriormente ser publicados em coletâneas impressas sob coordenação da Sustenere Publishing, da Companhia Brasileira de Produção Científica e seus parceiros autorizados. Os (as) autores (as) preservam os direitos autorais, mas não têm permissão para a publicação da contribuição em outro meio, impresso ou digital, em português ou em tradução. 